\title{
In situ Crystallization of Metallic Glass in an Atom Probe Instrument
}

\author{
David R. Diercks ${ }^{1 *}$, Rajesh Jha², Cristian V. Ciobanu ${ }^{2}$ and Aaron P. Stebner ${ }^{2}$ \\ 1. Department of Metallurgical and Materials Engineering, Colorado School of Mines, Golden, CO, \\ USA. \\ 2. Department of Mechanical Engineering, Colorado School of Mines, Golden, CO, USA. \\ * Corresponding Author: ddiercks@mines.edu
}

Amorphous metals and the thermal processing of them to produce nanocrystalline composites can lead to important magnetic [1] and mechanical [2] properties among other aspects. For these, control of the final size, composition, and distribution of the phases is of paramount importance. These can be influenced by small changes in initial compositions, temperature, and annealing time. Therefore, in seeking to modify the resulting multiphase alloys, it is key to understand not only the overall crystallization, but also rates of crystal nucleation and growth. Knowing what occurs at the earliest stages of the crystallization process would shed insight into how to modify the materials to control their properties. However, means of characterizing the early stages of crystallization are challenging because it happens on a time scale of picoseconds to seconds and a size scale of several atoms. In-situ synchrotron x-ray analysis has been used to capture the first seconds of crystallization for such alloys [3], but it provides results averaged on a several micrometer size scale. Atom probe tomography (APT) can examine individual clusters and has been applied to analysis of such materials, but for samples that were conventionally heated ex-situ for 5 to 60 minutes [4,5] or rapid thermal annealed for several seconds [6]. Recently it has been demonstrated that dynamic thermal processing can be performed inside a laser-pulsed atom probe instrument [7]. That is, the laser can be used to heat the specimen to induce a diffusive phase transformation as confirmed by transmission electron diffraction and subsequent atom probe analysis. With a laser pulse width of $\sim 10 \mathrm{ps}$ and such a small volume of material, the material is heated then quenched on time scales that are on the order of atomic motion. This is significant in that the earliest stages of crystallization may be captured.

Here, the results of the application of this in-situ laser heating method to crystallization of an amorphous FINEMET ${ }^{\circledR}\left(\mathrm{Fe}_{72.9} \mathrm{Cu}_{1} \mathrm{Nb}_{3.1} \mathrm{Si}_{16.2} \mathrm{~B}_{6.9}\right)$ alloy are presented. It is shown that the laser pulse energy can be correlated with an average peak temperature at the apex of the specimen. Laser pulsing at an energy which correspond to a temperature just below the known crystallization temperature of this alloy produces no measureable change, while laser pulsing at an energy which corresponds to a temperature above the crystallization temperature produces clear crystallization. Figure 1 shows a transmission electron microscopy dark-field image of one such specimen. Additionally, that image and selected area diffraction patterns collected down the shank of the specimen indicate the extent of the crystallization is limited to the top few hundred nanometers. This is significant in that it indicates the extent of the thermal gradient experienced along the specimen. Subsequent laser-pulsed APT analysis (at a much lower laser energy) through the depth of a laser annealed specimen demonstrated cluster size and cluster number density trends as a function of temperature consistent with what has been previously measured via APT from individually rapid thermal annealed FINEMET® alloys [6]. The main advantage here is that these trends were captured within a single specimen, saving significant specimen preparation and analysis time. Additionally, the estimated cumulative heating time experienced by the specimen during the laser annealing treatment was well under one second and could easily be reduced to under a microsecond. This means that this method can probe thermal treatment time-scales not easily captured by most other dynamic techniques. Taken together, in-situ thermal annealing via the laser in an atom probe instrument creates an opportunity for 
exploring sub-second annealing times and the effects of different temperatures on the resulting nm-scale composition distribution within a single specimen [8].

\section{References:}

[1] G Herzer, Acta Mater. 61 (2013), p. 718.

[2] CA Schuh, TC Hufnagel and U Ramamurty, Acta Mater. 55 (2007), p. 4067.

[3] U Köster et al., Mater. Sci. and Engin.: A 133 (1991), p. 611.

[4] K Hono et al., Acta Mater. 47 (1999), p. 997.

[5] M Ohnuma et al., J. Appl. Phys. 93 (2003), p. 9186.

[6] KG Pradeep et al., Acta Mater. 68 (2014), p. 295.

[7] R. Kirchhofer, D.R. Diercks, and B.P. Gorman, J. Mater. Res. 30 (2015), 1137-1147.

[8] This work is supported through the National Science Foundation "DMREF Collaborative Research: Accelerated Soft Magnetic Alloy Design and Synthesis Guided by Theory and Simulation," award number 1629026.
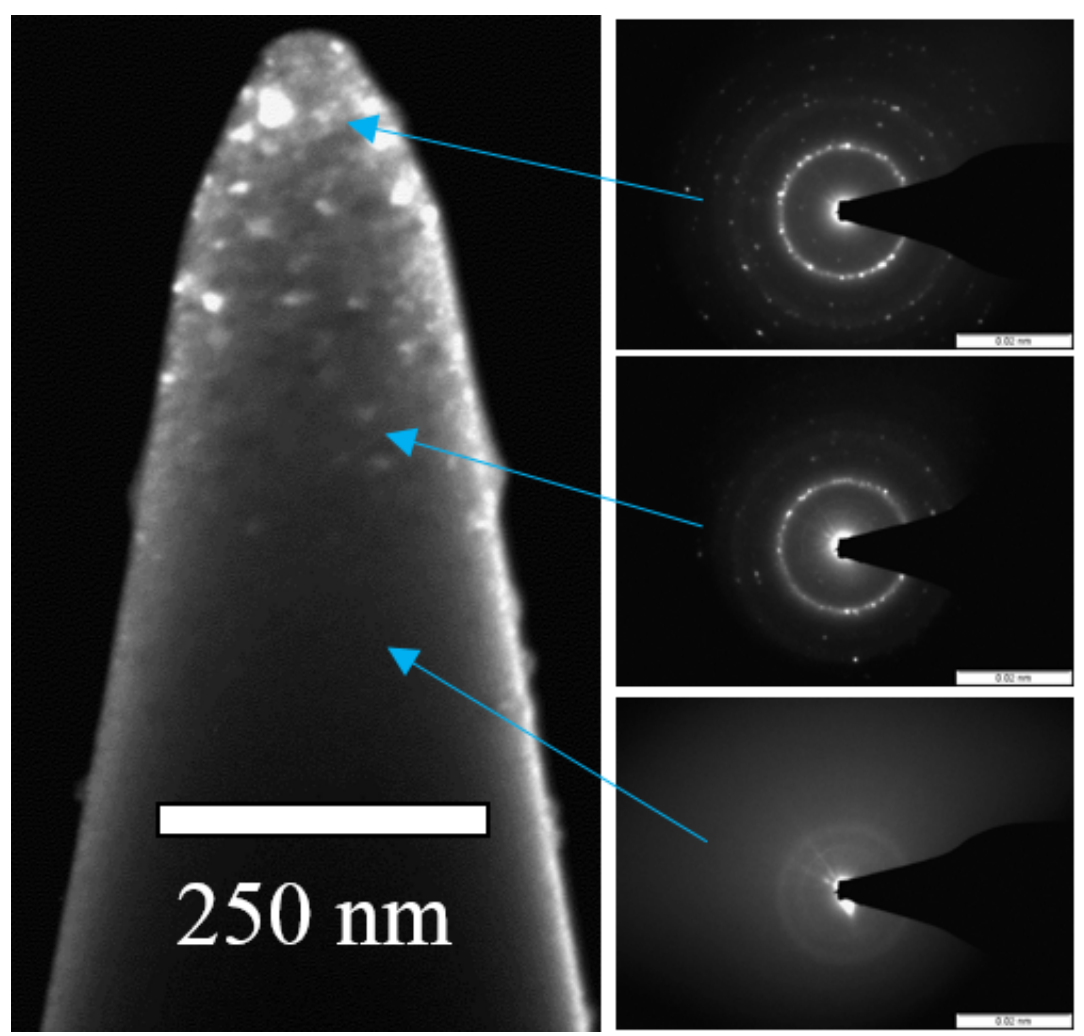

Figure 1. Dark-field transmission electron microscopy image of a FINEMET ${ }^{\circledR}$ alloy specimen thermally annealed via laser inside an atom probe instrument, inducing crystallization. The image along with selected area diffraction from the indicated locations show a transition from crystallized to uncrystallized material as a result of a thermal gradient along the specimen. 